Article

\title{
New Symmetric Differential and Integral Operators Defined in the Complex Domain
}

\author{
Rabha W. Ibrahim ${ }^{1, *,+([)}$ and Maslina Darus $2,+(\mathbb{C}$ \\ Cloud Computing Center, University Malaya, Kuala Lumpur 50603, Malaysia \\ 2 Center for Modelling and Data Science, Faculty of Science and Technology, Universiti Kebangsaan Malaysia, \\ Bangi 43600, Malaysia \\ * Correspondence: rabhaibrahim@yahoo.com \\ + These authors contributed equally to this work.
}

Received: 17 May 2019; Accepted: 2 July 2019; Published: 12 July 2019

\begin{abstract}
The symmetric differential operator is a generalization operating of the well-known ordinary derivative. These operators have advantages in boundary value problems, statistical studies and spectral theory. In this effort, we introduce a new symmetric differential operator (SDO) and its integral in the open unit disk. This operator is a generalization of the Sàlàgean differential operator. Our study is based on geometric function theory and its applications in the open unit disk. We formulate new classes of analytic functions using SDO depending on the symmetry properties. Moreover, we define a linear combination operator containing SDO and the Ruscheweyh derivative. We illustrate some inclusion properties and other inequalities involving SDO and its integral.
\end{abstract}

Keywords: univalent function; symmetric differential operator; unit disk; analytic function; subordination

MSC: 30C45

\section{Introduction}

Investigation of the theory of operators (differential, integral, mixed, convolution and linear) has been a capacity of apprehension for numerous scientists in all fields of mathematical sciences, such as mathematical physics, mathematical biology and mathematical computing. An additional definite field is the study of inequalities in the complex domain. Works' review shows masses of studies created by the classes of analytic functions. The relationship of geometry and analysis signifies a very central feature in geometric function theory in the open unit disk. This fast development is directly connected to the existence between analysis, construction and geometric performance [1]. In 1983, Sàlàgean introduced his famous differential operator of normalized analytic functions in the open unit disk [2]. This operator is generalized and extended to many classes of univalent functions. It plays a significant tool to develop the geometric structure of many analytic functions by suggesting different classes. Later this operator has been generalized and motivated by many researchers, for example, the Al-Oboudi differential operator [3]. Recently, a new study is presented by using the Sàlàgean operator [4]. Our research is to formulate a new symmetric differential operator and its integral by utilizing the concept of the symmetric derivative of complex variables. This concept is an operation, extending the original derivative. Note that its practical use in the the symmetry models in math modeling remains open. For example, for application in mathematical physics it is critical to employ group analysis methods. Such methods enable methods for branching solutions construction using group symmetry $[5,6]$. 


\section{Preparatory}

We shall need the following basic definitions throughout this paper. A function $\phi \in \Lambda$ is said to be univalent in $\mathbb{U}$ if it never takes the same value twice; that is, if $z_{1} \neq z_{2}$ in the open unit disk $\mathbb{U}=\{z \in \mathbb{C}:|z|<1\}$ then $\phi\left(z_{1}\right) \neq \phi\left(z_{2}\right)$ or equivalently, if $\phi\left(z_{1}\right)=\phi\left(z_{2}\right)$ then $z_{1}=z_{2}$. Without loss of generality, we can use the notion $\Lambda$ for our univalent functions taking the expansion

$$
\phi(z)=z+\sum_{n=2}^{\infty} \varphi_{n} z^{n}, \quad z \in \mathbb{U} .
$$

We let $\mathcal{S}$ denote the class of such functions $\phi \in \Lambda$ that are univalent in $\mathbb{U}$.

A function $\phi \in \mathcal{S}$ is said to be starlike with respect to origin in $\mathbb{U}$ if the linear segment joining the origin to every other point of $\phi(z:|z|=r<1)$ lies entirely in $\phi(z:|z|=r<1)$. In more picturesque language, the requirement is that every point of $\phi(z:|z|=r<1)$ be visible from the origin. A function $\phi \in \mathcal{S}$ is said to be convex in $\mathbb{U}$ if the linear segment joining any two points of $\phi(z:|z|=r<1)$ lies entirely in $\phi(z:|z|=r<1)$. In other words, a function $\phi \in \mathcal{S}$ is said to be convex in $\mathbb{U}$ if it is starlike with respect to each and every of its points. We denote the class of functions $\phi \in \mathcal{S}$ that are starlike with respect to origin by $\mathcal{S}^{*}$ and convex in $\mathbb{U}$ by $\mathcal{C}$.

Neatly linked to the classes $\mathcal{S}^{*}$ and $\mathcal{C}$ is the class $\mathcal{P}$ of all functions $\phi$ analytic in $\mathbb{U}$ and having positive real part in $\mathbb{U}$ with $\phi(0)=1$. In fact $f \in \mathcal{S}^{*}$ if and only if $z \phi^{\prime}(z) / \phi(z) \in \mathcal{P}$ and $\phi \in \mathcal{C}$ if and only if $1+z \phi^{\prime \prime}(z) / \phi^{\prime}(z) \in \mathcal{P}$. In general, for $\epsilon \in[0,1)$ we let $\mathcal{P}(\epsilon)$ consist of functions $\phi$ analytic in $\mathbb{U}$ with $\phi(0)=1$ so that $\Re(\phi(z))>\epsilon$ (' $\Re^{\prime}$ represents to the real part) for all $z \in \mathbb{U}$. Note that $\mathcal{P}\left(\epsilon_{2}\right) \subset \mathcal{P}\left(\epsilon_{1}\right) \subset \mathcal{P}(0) \equiv \mathcal{P}$ for $0<\epsilon_{1}<\epsilon_{2}$ (e.g., see Duren [1]).

For functions $\phi$ and $\psi$ in $\Lambda$ we say that $\phi$ is subordinate to $\psi$, denoted by $\phi \prec \psi$, if there exists a Schwarz function $\omega$ with $\omega(0)=0$ and $|\omega(z)|<1$ so that $\phi(z)=\psi(\omega(z))$ for all $z \in \mathbb{U}$ (see [7]). Evidently $\phi(z) \prec \psi(z)$ is equivalent to $\phi(0)=\psi(0)$ and $\phi(\mathbb{U}) \subset \psi(\mathbb{U})$. We request the following results, which can be located in [7].

Lemma 1. For $a \in \mathbb{C}$ and positive integer $n$ let $\mathfrak{H}[a, n]=\left\{\varrho: \varrho(z)=a+a_{n} z^{n}+a_{n+1} z^{n+1}+\ldots\right\}$.

i. If $\gamma \in \mathbb{R}$ then $\Re\left(\varrho(z)+\gamma z \varrho^{\prime}(z)\right)>0 \Longrightarrow \Re(\varrho(z))>0$. Moreover, if $\gamma>0$ and $\varrho \in \mathfrak{H}[1, n]$, then there are constants $\lambda>0$ and $\beta>0$ with $\beta=\beta(\gamma, \lambda, n)$ so that

$$
\varrho(z)+\gamma z \varrho^{\prime}(z) \prec\left[\frac{1+z}{1-z}\right]^{\beta} \Rightarrow \varrho(z) \prec\left[\frac{1+z}{1-z}\right]^{\lambda} .
$$

ii. If $\delta \in[0,1)$ and $\varrho \in \mathfrak{H}[1, n]$ then there is a constant $\lambda>0$ with $\lambda=\lambda(\alpha, n)$ so that

$$
\Re\left(\varrho^{2}(z)+2 \varrho(z) \cdot z \varrho^{\prime}(z)\right)>\delta \Rightarrow \Re(\varrho(z))>\lambda .
$$

iii. If $\varrho \in \mathfrak{H}[a, n]$ with $\Re a>0$ then $\Re\left(\varrho(z)+z \varrho^{\prime}(z)+z^{2} \varrho^{\prime \prime}(z)\right)>0$ or for $\vartheta: \mathbb{U} \rightarrow \mathbb{R}$ with $\Re\left(\varrho(z)+\vartheta(z) \frac{z \varrho^{\prime}(z)}{\varrho(z)}\right)>0$ then $\Re(\varrho(z))>0$.

Lemma 2. Let $h$ be a convex function with $h(0)=a$, and let $\mu \in \mathbb{C} \backslash\{0\}$ be a complex number with $\Re \gamma \geq 0$. If $\varrho \in \mathfrak{H}[a, n]$, and $\varrho(z)+(1 / \mu) z \varrho^{\prime}(z) \prec h(z), \quad z \in U$, then $\varrho(z) \prec \iota(z) \prec h(z)$, where

$$
\iota(z)=\frac{\mu}{n z^{\mu / n}} \int_{0}^{z} h(t) t^{\frac{\mu}{(n-1)}} d t, \quad z \in U .
$$




\section{Formulas of Symmetric Operators}

Let $\phi \in \Lambda$, taking the power series (1). For a function $\phi(z)$ and a constant $\alpha \in[0,1]$, we formulate the SDO as follows:

$$
\begin{aligned}
\mathcal{M}_{\alpha}^{0} \phi(z) & =\phi(z) \\
\mathcal{M}_{\alpha}^{1} \phi(z) & =\alpha z \phi^{\prime}(z)-(1-\alpha) z \phi^{\prime}(-z) \\
& =\alpha\left(z+\sum_{n=2}^{\infty} n \varphi_{n} z^{n}\right)-(1-\alpha)\left(-z+\sum_{n=2}^{\infty} n(-1)^{n} \varphi_{n} z^{n}\right) \\
& =z+\sum_{n=2}^{\infty}\left[n\left(\alpha-(1-\alpha)(-1)^{n}\right)\right] \varphi_{n} z^{n} \\
\mathcal{M}_{\alpha}^{2} \phi(z) & =\mathcal{M}_{\alpha}^{1}\left[\mathcal{M}_{\alpha}^{1} \phi(z)\right]=z+\sum_{n=2}^{\infty}\left[n\left(\alpha-(1-\alpha)(-1)^{n}\right)\right]^{2} \varphi_{n} z^{n} \\
\vdots & \mathcal{M}_{\alpha}^{k} \phi(z)
\end{aligned}
$$

It is clear that when $\alpha=1$, we have Sàlàgean differential operator [2] $\mathcal{S}^{k} \phi(z)=z+\sum_{n=2}^{\infty} n^{k} \varphi_{n} z^{n}$. We may say that SDO (2) is the symmetric Sàlàgean differential operator in the open unit disk. In the same manner of the formula of Sàlàgean integral operator, we consume that for a function $\phi \in \Lambda$, the symmetric integral operator $\mathcal{J}_{\alpha}^{k}$ satisfies

$$
\mathcal{J}_{\alpha}^{k} \phi(z)=z+\sum_{n=2}^{\infty} \frac{1}{\left[n\left(\alpha-(1-\alpha)(-1)^{n}\right)\right]^{k}} \varphi_{n} z^{n} \in \Lambda .
$$

Similarly, when $\alpha=1$, we have Sàlàgean integral operator [2], Remark 5. Furthermore, we conclude the relation $\mathcal{M}_{\alpha}^{k}\left(\mathcal{J}_{\alpha}^{k} \phi(z)\right)=\mathcal{J}_{\alpha}^{k}\left(\mathcal{M}_{\alpha}^{k} \phi(z)\right)=\phi(z)$.

Next, we proceed to formulate a linear combination operator involving SDO and the Ruscheweyh derivative. For a function $\phi \in \Lambda$, the Ruscheweyh derivative achieves the formula

$$
\mathcal{R}^{k} \phi(z)=z+\sum_{n=2}^{\infty} C_{k+n-1}^{k} \varphi_{n} z^{n}
$$

where the term $C_{k+n-1}^{k}$ is the combination coefficients. In this note, we introduce a new operator combining $R^{k}$ and $\mathcal{M}_{\alpha}^{k}$ as follows:

$$
\begin{aligned}
\mathbf{C}_{\alpha, \kappa}^{k} \phi(z) & =(1-\kappa) \mathcal{R}^{k} \phi(z)+\kappa \mathcal{M}_{\alpha}^{k} \phi(z) \\
& =z+\sum_{n=2}^{\infty}\left((1-\kappa) C_{k+n-1}^{k}+\kappa\left[n\left(\alpha-(1-\alpha)(-1)^{n}\right)\right]^{k}\right) \varphi_{n} z^{n} .
\end{aligned}
$$

\section{Remark 1.}

- $\quad k=0 \Longrightarrow \mathbf{C}_{\alpha, \kappa}^{0} \phi(z)=\phi(z)$;

- $\quad \alpha=1 \Longrightarrow \mathbf{C}_{1, k}^{k} \phi(z)=\mathcal{L}_{\kappa}^{k} \phi(z) ;[8]$ (Lupas operator)

- $\quad \kappa=0 \Longrightarrow \mathbf{C}_{\alpha, 0}^{k} \phi(z)=\mathcal{R}^{k} \phi(z)$;

- $\alpha=1, \kappa=1 \Longrightarrow \mathbf{C}_{1,1}^{k} \phi(z)=\mathcal{S}^{k} \phi(z)$;

- $\quad \kappa=1 \Longrightarrow \mathbf{C}_{\alpha, \kappa}^{k} \phi(z)=\mathcal{M}_{\alpha}^{k} \phi(z)$. 
We shall deal with the following classes

$$
S_{k}^{* \alpha}(h)=\left\{\phi \in \Lambda: \frac{z\left(\mathcal{M}_{\alpha}^{k} \phi(z)\right)^{\prime}}{\mathcal{M}_{\alpha}^{k} \phi(z)} \prec h(z), h \in \mathcal{C}\right\} .
$$

Obviously, the subclass $S_{0}^{*}(h)=\mathcal{S}^{*}(h)$.

Definition 1. If $\phi \in \Lambda$, then $\phi \in \mathbb{J}_{\alpha}^{b}(A, B, k)$ if and only if

$$
\begin{gathered}
1+\frac{1}{b}\left(\frac{2 \mathcal{M}_{\alpha}^{k+1} \phi(z)}{\mathcal{M}_{\alpha}^{k} \phi(z)-\mathcal{M}_{\alpha}^{k} \phi(-z)}\right) \prec \frac{1+A z}{1+B z}, \\
(z \in \mathbb{U},-1 \leq B<A \leq 1, k=1,2, \ldots, b \in \mathbb{C} \backslash\{0\}, \alpha \in[0,1]) .
\end{gathered}
$$

- $\alpha=1 \Longrightarrow[9]$

- $\alpha=1, B=0 \Longrightarrow[10]$

- $\alpha=1, A=1, B=-1, b=2 \Longrightarrow[11]$.

Definition 2. Let $\epsilon \in[0,1), \alpha \in[0,1], \kappa \geq 0$, and $k \in \mathbb{N}$. A function $\phi \in \Lambda$ is said to be in the set $T_{k}(\alpha, \kappa, \epsilon)$ if and only if

$$
\Re\left(\left(\mathbf{C}_{\alpha, \kappa}^{k} \phi(z)\right)^{\prime}\right)>\epsilon, \quad z \in U .
$$

\section{Geometric Results}

In this section, we utilize the above constructions of the symmetric operators to get some geometric fulfillment.

Theorem 1. For $\phi \in \Lambda$ if one of the following facts holds

- $\quad$ The operator $\mathcal{M}_{\alpha}^{k} \phi(z)$ in (2) is of bounded boundary rotation;

- $\quad \phi$ achieves the subordination inequality

$$
\left(\mathcal{M}_{\alpha}^{k} \phi(z)\right)^{\prime} \prec\left(\frac{1+z}{1-z}\right)^{\beta}, \quad \beta>0, z \in \mathbb{U}, \quad \alpha \in[0, \infty) ;
$$

- $\quad f$ satisfies the inequality

$$
\Re\left(\left(\mathcal{M}_{\alpha}^{k} \phi(z)\right)^{\prime} \frac{\mathcal{M}_{\alpha}^{k} \phi(z)}{z}\right)>\frac{\delta}{2}, \quad \delta \in[0,1), z \in \mathbb{U},
$$

- $\quad \phi$ admits the inequality

$$
\left.\left.\Re\left(z \mathcal{M}_{\alpha}^{k} \phi(z)\right)^{\prime \prime}-\mathcal{M}_{\alpha}^{k} \phi(z)\right)^{\prime}+2 \frac{\left.\mathcal{M}_{\alpha}^{k} \phi(z)\right)}{z}\right)>0,
$$

- $\quad \phi$ confesses the inequality

$$
\Re\left(\frac{\left.z \mathcal{M}_{\alpha}^{k} \phi(z)\right)^{\prime}}{\left.\mathcal{M}_{\alpha}^{k} \phi(z)\right)}+2 \frac{\mathcal{M}_{\alpha}^{k} \phi(z)}{z}\right)>1
$$

then $\frac{\mathcal{M}_{\alpha}^{k} \phi(z)}{z} \in \mathcal{P}(\epsilon)$ for some $\epsilon \in[0,1)$. 
Proof. Define a function $\varrho$ as follows

$$
\varrho(z)=\frac{\mathcal{M}_{\alpha}^{k} \phi(z)}{z} \Rightarrow z \varrho^{\prime}(z)+\varrho(z)=\left(\mathcal{M}_{\alpha}^{k} \phi(z)\right)^{\prime} .
$$

By the first fact, $\mathcal{M}_{\alpha}^{k} \phi(z)$ is of bounded boundary rotation, it implies that $\Re\left(z \varrho^{\prime}(z)+\varrho(z)\right)>0$. Thus, by Lemma 1.i, we obtain $\Re(\varrho(z))>0$ which yields the first part of the theorem.

In view of the second fact, we have the following subordination relation

$$
\left(\mathcal{M}_{\alpha}^{k} \phi(z)\right)^{\prime}=z \varrho^{\prime}(z)+\varrho(z) \prec\left[\frac{1+z}{1-z}\right]^{\beta} .
$$

Now, according to Lemma 1.i, there is a constant $\gamma>0$ with $\beta=\beta(\gamma)$ such that

$$
\frac{\mathcal{M}_{\alpha}^{k} \phi(z)}{z} \prec\left(\frac{1+z}{1-z}\right)^{\gamma}
$$

This implies that $\Re\left(\mathcal{M}_{\alpha}^{k} \phi(z) / z\right)>\epsilon$, for some $\epsilon \in[0,1)$.

Finally, consider the third fact, a simple computation yields

$$
\Re\left(\varrho^{2}(z)+2 \varrho(z) \cdot z \varrho^{\prime}(z)\right)=2 \Re\left(\left(\mathcal{M}_{\alpha}^{k} \phi(z)\right)^{\prime} \frac{\mathcal{M}_{\alpha}^{k} \phi(z)}{z}\right)>\delta .
$$

In virtue of Lemma 1.ii, there is a constant $\lambda>0$ such that $\Re(\rho(z))>\lambda$ which implies that $\varrho(z)=\frac{\mathcal{M}_{\alpha}^{k} \phi(z)}{z} \in \mathcal{P}(\epsilon)$ for some $\epsilon \in[0,1)$. It follows from (5) that $\left.\Re\left(\mathcal{M}_{\alpha}^{k} \phi(z)\right)^{\prime}\right)>0$ and thus by Noshiro-Warschawski and Kaplan Theorems, $\mathcal{M}_{\alpha}^{k} \phi(z)$ is univalent and of bounded boundary rotation in $\mathbb{U}$.

By differentiating (4) and taking the real, we have

$$
\Re\left(\varrho(z)+z \varrho^{\prime}(z)+z^{2} \varrho^{\prime \prime}(z)\right)=\Re\left(z\left(\mathcal{M}_{\alpha}^{k} \phi(z)\right)^{\prime \prime}-\left(\mathcal{M}_{\alpha}^{k} \phi(z)\right)^{\prime}+2 \frac{\mathcal{M}_{\alpha}^{k} \phi(z)}{z}\right)>0 .
$$

Thus, in virtue of Lemma 1.ii, we obtain $\Re\left(\frac{\mathcal{M}_{\alpha}^{k} \phi(z)}{z}\right)>0$.

By logarithmic differentiation (4) and taking the real, we have

$$
\Re\left(\varrho(z)+\frac{z \varrho^{\prime}(z)}{\varrho(z)}+z^{2} \varrho^{\prime \prime}(z)\right)=\Re\left(\frac{z\left(\mathcal{M}_{\alpha}^{k} \phi(z)\right)^{\prime}}{\mathcal{M}_{\alpha}^{k} \phi(z)}+2 \frac{\mathcal{M}_{\alpha}^{k} \phi(z)}{z}-1\right)>0 .
$$

Hence, in virtue of Lemma 1.iii, with $\vartheta(z)=1$, we conclude that $\Re\left(\frac{\mathcal{M}_{\alpha}^{k} \phi(z)}{z}\right)>0$. This completes the proof.

Theorem 2. Let $\phi \in S_{k}^{* \alpha}(h)$, where $h(z)$ is convex univalent function in $\mathbb{U}$. Then

$$
\mathcal{M}_{\alpha}^{k} \phi(z) \prec z \exp \left(\int_{0}^{z} \frac{h(\omega(\xi))-1}{\xi} d \xi\right),
$$

where $\omega(z)$ is analytic in $\mathbb{U}$, with $\omega(0)=0$ and $|\omega(z)|<1$. Furthermore, for $|z|=\eta, \mathcal{M}_{\alpha}^{k} \phi(z)$ achieves the inequality

$$
\exp \left(\int_{0}^{1} \frac{h(\omega(-\eta))-1}{\eta}\right) d \eta \leq\left|\frac{\mathcal{M}_{\alpha}^{k} \phi(z)}{z}\right| \leq \exp \left(\int_{0}^{1} \frac{h(\omega(\eta))-1}{\eta}\right) d \eta .
$$


Proof. Since $\phi \in S_{k}^{* \alpha}(h)$, we have

$$
\left(\frac{z\left(\mathcal{M}_{\alpha}^{k} \phi(z)\right)^{\prime}}{\mathcal{M}_{\alpha}^{k} \phi(z)}\right) \prec h(z), \quad z \in \mathbb{U},
$$

which means that there exists a Schwarz function with $\omega(0)=0$ and $|\omega(z)|<1$ such that

$$
\left(\frac{z\left(\mathcal{M}_{\alpha}^{k} \phi(z)\right)^{\prime}}{\mathcal{M}_{\alpha}^{k} \phi(z)}\right)=h(\omega(z)), \quad z \in \mathbb{U},
$$

which implies that

$$
\left(\frac{\left(\mathcal{M}_{\alpha}^{k} \phi(z)\right)^{\prime}}{\mathcal{M}_{\alpha}^{k} \phi(z)}\right)-\frac{1}{z}=\frac{h(\omega(z))-1}{z} .
$$

Integrating both sides, we have

$$
\log \mathcal{M}_{\alpha}^{k} \phi(z)-\log z=\int_{0}^{z} \frac{h(\omega(\xi))-1}{\xi} d \xi .
$$

Consequently, this yields

$$
\log \frac{\mathcal{M}_{\alpha}^{k} \phi(z)}{z}=\int_{0}^{z} \frac{h(\omega(\xi))-1}{\xi} d \xi .
$$

By using the definition of subordination, we get

$$
\mathcal{M}_{\alpha}^{k} \phi(z) \prec z \exp \left(\int_{0}^{z} \frac{h(\omega(\xi))-1}{\xi} d \xi\right) .
$$

In addition, we note that the function $h(z)$ maps the disk $0<|z|<\eta<1$ onto a region which is convex and symmetric with respect to the real axis, that is

$$
h(-\eta|z|) \leq \Re(h(\omega(\eta z))) \leq h(\eta|z|), \quad \eta \in(0,1)
$$

which yields the following inequalities:

$$
h(-\eta) \leq h(-\eta|z|), \quad h(\eta|z|) \leq h(\eta)
$$

and

$$
\int_{0}^{1} \frac{h(\omega(-\eta|z|))-1}{\eta} d \eta \leq \Re\left(\int_{0}^{1} \frac{h(\omega(\eta))-1}{\eta} d \eta\right) \leq \int_{0}^{1} \frac{h(\omega(\eta|z|))-1}{\eta} d \eta .
$$

By using the above relations and Equation (6), we conclude that

$$
\int_{0}^{1} \frac{h(\omega(-\eta|z|))-1}{\eta} d \eta \leq \log \left|\frac{\mathcal{M}_{\alpha}^{k} \phi(z)}{z}\right| \leq \int_{0}^{1} \frac{h(\omega(\eta|z|))-1}{\eta} d \eta .
$$

This equivalence to the inequality

$$
\exp \left(\int_{0}^{1} \frac{h(\omega(-\eta|z|))-1}{\eta} d \eta\right) \leq\left|\frac{\mathcal{M}_{\alpha}^{k} \phi(z)}{z}\right| \leq \exp \left(\int_{0}^{1} \frac{h(\omega(\eta|z|))-1}{\eta} d \eta\right) .
$$

Thus, we obtain

$$
\exp \left(\int_{0}^{1} \frac{h(\omega(-\eta))-1}{\eta}\right) d \eta \leq\left|\frac{\mathcal{M}_{\alpha}^{k} \phi(z)}{z}\right| \leq \exp \left(\int_{0}^{1} \frac{h(\omega(\eta))-1}{\eta}\right) d \eta .
$$


This completes the proof.

Theorem 3. Consider the class $\mathbb{J}_{\alpha}^{b}(A, B, k)$ in Definition 1. If $\phi \in \mathbb{J}_{\alpha}^{b}(A, B, k)$ then the odd function

$$
\mathfrak{O}(z)=\frac{1}{2}[\phi(z)-\phi(-z)], \quad z \in \mathbb{U}
$$

achieves the following inequality

$$
1+\frac{1}{b}\left(\frac{\mathcal{M}_{\alpha}^{k+1} \mathfrak{O}(z)}{\mathcal{M}_{\alpha}^{k} \mathfrak{O}(z)}-1\right) \prec \frac{1+A z}{1+B z}
$$

and

$$
\begin{gathered}
\Re\left(\frac{z \mathfrak{O}(z)^{\prime}}{\mathfrak{O}(z)}\right) \geq \frac{1-r^{2}}{1+r^{2}}, \quad|z|=r<1, \\
(z \in \mathbb{U},-1 \leq B<A \leq 1, k=1,2, \ldots, b \in \mathbb{C} \backslash\{0\}, \alpha \in[0,1]) .
\end{gathered}
$$

Proof. Since $\phi \in \mathbb{J}_{\alpha}^{b}(A, B, k)$ then there is a function $P \in \mathbb{J}(A, B)$ such that

$$
b(P(z)-1)=\left(\frac{2 \mathcal{M}_{\alpha}^{k+1} \phi(z)}{\mathcal{M}_{\alpha}^{k} \phi(z)-\mathcal{M}_{\alpha}^{k} \phi(-z)}\right)
$$

and

$$
b(P(-z)-1)=\left(\frac{-2 \mathcal{M}_{\alpha}^{k+1} \phi(-z)}{\mathcal{M}_{\alpha}^{k} \phi(z)-\mathcal{M}_{\alpha}^{k} \phi(-z)}\right) .
$$

This implies that

$$
1+\frac{1}{b}\left(\frac{\mathcal{M}_{\alpha}^{k+1} \mathfrak{O}(z)}{\mathcal{M}_{\alpha}^{k} \mathfrak{O}(z)}-1\right)=\frac{P(z)+P(-z)}{2} .
$$

Also, since

$$
P(z) \prec \frac{1+A z}{1+B z}
$$

where $\frac{1+A z}{1+B z}$ is univalent then by the definition of the subordination, we obtain

$$
1+\frac{1}{b}\left(\frac{\mathcal{M}_{\alpha}^{k+1} \mathfrak{O}(z)}{\mathcal{M}_{\alpha}^{k} \mathfrak{O}(z)}-1\right) \prec \frac{1+A z}{1+B z} .
$$

Moreover, the function $\mathfrak{O}(z)$ is starlike in $\mathbb{U}$ which implies that

$$
\frac{z \mathfrak{O}(z)^{\prime}}{\mathfrak{O}(z)} \prec \frac{1-z^{2}}{1+z^{2}}
$$

that is, there exists a Schwarz function $\wp \in \mathbb{U},|\wp(z)| \leq|z|<1, \wp(0)=0$ such that

$$
\Phi(z):=\frac{z \mathfrak{O}(z)^{\prime}}{\mathfrak{D}(z)} \prec \frac{1-\wp(z)^{2}}{1+\wp(z)^{2}}
$$

which yields that there is $\xi,|\xi|=r<1$ such that

$$
\wp^{2}(\xi)=\frac{1-\Phi(\xi)}{1+\Phi(\xi)}, \quad \xi \in \mathbb{U} .
$$


A calculation gives that

$$
\left|\frac{1-\Phi(\xi)}{1+\Phi(\xi)}\right|=|\wp(\xi)|^{2} \leq|\xi|^{2} .
$$

Hence, we have the following conclusion

$$
\left|\Phi(\xi)-\frac{1+|\xi|^{4}}{1-|\xi|^{4}}\right|^{2} \leq \frac{4|\xi|^{4}}{\left(1-|\xi|^{4}\right)^{2}}
$$

or

$$
\left|\Phi(z)-\frac{1+|\xi|^{4}}{1-|\xi|^{4}}\right| \leq \frac{2|\xi|^{2}}{\left(1-|\xi|^{4}\right)}
$$

This implies that

$$
\Re(\Phi(z)) \geq \frac{1-r^{2}}{1+r^{2}}, \quad|\xi|=r<1 .
$$

Next consequence result of Theorem 3 can be found in $[9,11]$ respectively.

Corollary 1. Let $\alpha=1$ in Theorem 3. Then

$$
1+\frac{1}{b}\left(\frac{\mathcal{M}_{1}^{k+1} \mathfrak{O}(z)}{\mathcal{M}_{1}^{k} \mathfrak{O}(z)}-1\right) \prec \frac{1+A z}{1+B z} .
$$

Corollary 2. Let $\alpha=1, k=1$ in Theorem 3. Then

$$
1+\frac{1}{b}\left(\frac{\mathcal{M}_{1}^{2} \mathfrak{O}(z)}{\mathcal{M}_{1} \mathfrak{O}(z)}-1\right) \prec \frac{1+A z}{1+B z} .
$$

Theorem 4. The set $T_{k}(\alpha, \kappa, \epsilon)$ in Definition 2 is convex.

Proof. Let $\phi_{i}, i=1,2$ be two functions in the set $T_{k}(\alpha, \kappa, \epsilon)$ satisfying $\phi_{1}(z)=z+\sum_{n=2}^{\infty} a_{n} z^{n}$ and $\phi_{2}(z)=z+\sum_{n=2}^{\infty} b_{n} z^{n}$. It is sufficient to prove that the function

$$
H(z)=c_{1} \phi_{1}(z)+c_{2} \phi_{2}(z), \quad z \in \mathbb{U}
$$

is in $T_{k}(\alpha, \kappa, \epsilon)$, where $c_{1}>0, c_{2}>0$ and $c_{1}+c_{2}=1$. By the definition of $H(z)$, a calculation implies that

$$
H(z)=z+\sum_{n=2}^{\infty}\left(c_{1} a_{n}+c_{2} b_{n}\right) z^{n}
$$

then under the operator $\mathbf{C}_{\alpha, \kappa}^{k}$, we obtain

$$
\begin{gathered}
\mathbf{C}_{\alpha, k}^{k} H(z)=z+\sum_{n=2}^{\infty}\left(c_{1} a_{n}+c_{2} b_{n}\right) \\
\times\left[(1-\kappa) C_{k+n-1}^{k}+\kappa\left(n\left[\alpha-(1-\alpha)(-1)^{n}\right]\right)^{k}\right] z^{n} .
\end{gathered}
$$


By taking the derivative for the last equation and following by the real, we have

$$
\begin{aligned}
& \Re\left\{\left(\mathbf{C}_{\alpha, \kappa}^{k} H(z)\right)^{\prime}\right\} \\
& =1+c_{1} \Re\left\{\sum_{n=2}^{\infty} n\left[(1-\kappa) C_{k+n-1}^{k}+\kappa\left(n\left[\alpha-(1-\alpha)(-1)^{n}\right]\right)^{k} a_{n} z^{n-1}\right\}\right. \\
& +c_{2} \Re\left\{\sum_{n=2}^{\infty} n\left[(1-\kappa) C_{k+n-1}^{k}+\kappa\left(n\left[\alpha-(1-\alpha)(-1)^{n}\right]\right)^{k} b_{n} z^{n-1}\right\}\right. \\
& >1+c_{1}(\epsilon-1)+c_{2}(\epsilon-1) \\
& =\epsilon .
\end{aligned}
$$

This completes the proof.

Next consequence result of Theorem 4 can be found in [8].

Corollary 3. Let $\alpha=1$ in Theorem 4 . Then the set $T_{k}(1, \kappa, \epsilon)$ is convex.

Theorem 5. Let $\phi \in T_{k}(\alpha, \kappa, \epsilon)$, and let $\varphi$ be convex. Then for a function

$$
F(z)=\frac{2+c}{z^{1+c}} \int_{0}^{z} t^{c} \phi(t) d t, \quad z \in U
$$

the subordination

$$
\left(\mathbf{C}_{\alpha, k}^{k} \phi(z)\right)^{\prime} \prec \varphi(z)+\frac{\left(z \varphi^{\prime}(z)\right)}{2+c}, \quad c>0,
$$

implies

$$
\left(\mathbf{C}_{\alpha, \kappa}^{k} F(z)\right)^{\prime} \prec \varphi(z),
$$

and this result is sharp.

Proof. Our aim is to apply Lemma 2. By the definition of $F(z)$, we obtain

$$
\left(\mathbf{C}_{\alpha, k}^{k} F(z)\right)^{\prime}+\frac{\left(\mathbf{C}_{\alpha, \kappa}^{k} F(z)\right)^{\prime \prime}}{2+c}=\left(\mathbf{C}_{\alpha, k}^{k} \phi(z)\right)^{\prime} .
$$

By the assumption, we get

$$
\left(\mathbf{C}_{\alpha, \kappa}^{k} F(z)\right)^{\prime}+\frac{\left(\mathbf{C}_{\alpha, \kappa}^{k} F(z)\right)^{\prime \prime}}{2+c} \prec \varphi(z)+\frac{\left(z \varphi^{\prime}(z)\right)}{2+c} .
$$

By letting

$$
\varrho(z):=\left(\mathbf{C}_{\alpha, \kappa}^{k} F(z)\right)^{\prime},
$$

one can find

$$
\varrho(z)+\frac{\left(z \varrho^{\prime}(z)\right)}{2+c} \prec \varphi(z)+\frac{\left(z \varphi^{\prime}(z)\right)}{2+c} .
$$

In virtue of Lemma 2, we have

$$
\left(\mathbf{C}_{\alpha, \kappa}^{k} F(z)\right)^{\prime} \prec \varphi(z),
$$

and $\varphi$ is the best dominant. 
Theorem 6. Let $\varphi$ be convex achieving $\varphi(0)=1$. If

$$
\left(\mathbf{C}_{\alpha, \kappa}^{k} \phi(z)\right)^{\prime} \prec \varphi(z)+z \varphi^{\prime}(z), \quad z \in U,
$$

then

$$
\frac{\mathbf{C}_{\alpha, \kappa}^{k} \phi(z)}{z} \prec \varphi(z),
$$

and this result is sharp.

Proof. Our aim is to apply Lemma 1. Define the function

$$
\varrho(z):=\frac{\mathbf{C}_{\alpha, k}^{k} \phi(z)}{z} \in \mathfrak{H}[1,1]
$$

By this assumption, yields

$$
\mathbf{C}_{\alpha, k}^{k} \phi(z)=z \varrho(z) \Longrightarrow\left(\mathbf{C}_{\alpha, \kappa}^{k} \phi(z)\right)^{\prime}=\varrho(z)+z \varrho^{\prime}(z) .
$$

Thus, we deduce the following subordination:

$$
\varrho(z)+z \varrho^{\prime}(z) \prec \varphi(z)+z \varphi^{\prime}(z) .
$$

In view of Lemma 1, we receive

$$
\frac{\mathbf{C}_{\alpha, \kappa}^{k} \phi(z)}{z} \prec \varphi(z),
$$

and $\varphi$ is the best dominant.

Theorem 7. If $\phi \in \Lambda$ satisfies the subordination relation

$$
\left(\mathbf{C}_{\alpha, \kappa}^{k} \phi(z)\right)^{\prime} \prec\left(\frac{1+z}{1-z}\right)^{\beta}, \quad z \in \mathbb{U}, \beta>0,
$$

then

$$
\Re\left(\frac{\mathbf{C}_{\alpha, \kappa}^{k} \phi(z)}{z}\right)>\epsilon
$$

for some $\epsilon \in[0,1)$.

Proof. Define a function $\varrho$ as in (7). Then, by subordination properties, we have

$$
\left(\mathbf{C}_{\alpha, \kappa}^{k} \phi(z)\right)^{\prime}=z \varrho^{\prime}(z)+\varrho(z) \prec\left[\frac{1+z}{1-z}\right]^{\beta} .
$$

Now, in view of Lemma 1.i, there is a constant $\gamma>0$ with $\beta=\beta(\gamma)$ such that

$$
\frac{\mathrm{C}_{\alpha, k}^{k} \phi(z)}{z} \prec\left(\frac{1+z}{1-z}\right)^{\gamma}
$$

This implies that $\Re\left(\mathbf{C}_{\alpha, k}^{k} \phi(z) / z\right)>\epsilon$, for some $\epsilon \in[0,1)$.

Theorem 8. If $\phi \in \Lambda$ satisfies the inequality

$$
\Re\left(\left(\mathbf{C}_{\alpha, k}^{k} \phi(z)\right)^{\prime} \frac{\mathbf{C}_{\alpha, k}^{k} \phi(z)}{z}\right)>\frac{\alpha}{2}, \quad z \in U, \alpha \in[0,1)
$$


then $\mathbf{C}_{\alpha, \kappa}^{k} \phi(z) \in T_{k}(\alpha, \kappa, \epsilon)$ for some $\epsilon \in[0,1)$. Furthermore, it is univalent and of bounded boundary rotation in $U$.

We inform the readers that in virtue of Noshiro-Warschawski Theorem (Duren [1], p. 47) if a function $\phi$ is analytic in the simply connected complex domain $\mathbb{U}$ and $\Re\left\{\phi^{\prime}(z)\right\}>0$ in $\mathbb{U}$ then $\phi$ is univalent in $\mathbb{U}$ and in view of Kaplan's Theorem (Duren [1], p. 48) such functions $\phi$ is of bounded boundary rotation.

Proof. Define a function $\varrho$ as in (7). A simple computation yields

$$
\left.\Re\left(\varrho^{2}(z)+2 \varrho(z) \cdot z \varrho^{\prime}(z)\right)=2 \Re\left(\mathbf{C}_{\alpha, \kappa}^{k} \phi(z)\right)^{\prime} \frac{\mathbf{C}_{\alpha, k}^{k} \phi(z)}{z}\right)>\alpha .
$$

By virtue of Lemma 1.ii, there is a constant $\lambda$ depending on $\alpha$ such that $\Re(\varrho(z))>\lambda$, which implies that $\Re(\varrho(z))>\epsilon$ for some $\epsilon \in[0,1)$. It follows from (8) that $\left.\Re\left(\mathbf{C}_{\alpha, k}^{k} \phi(z)\right)^{\prime}\right)>\epsilon$ and thus by Noshiro-Warschawski and Kaplan Theorems, $\mathrm{C}_{\alpha, k}^{k} \phi(z)$ is univalent and of bounded boundary rotation in $\mathbb{U}$.

Example 1. We have the following data: $\phi(z)=z /(1-z), \alpha=0.25$. A calculation brings

$$
\begin{aligned}
\mathcal{M}_{\alpha}^{1} \phi(z) & =\alpha z \phi^{\prime}(z)-(1-\alpha) z \phi^{\prime}(-z) \\
& =\frac{0.25 z}{(1-z)^{2}}+\frac{0.75 z}{(1+z)^{2}}=\frac{z\left(z^{2}-z+1\right)}{(1-z)^{2}(1+z)^{2}} \\
& =z-z^{2}+3 z^{3}-2 z^{4}+5 z^{5}+o\left(z^{6}\right)
\end{aligned}
$$

with

$$
\begin{aligned}
& \Re\left(\left(\mathcal{M}_{\alpha}^{1} \phi(z)\right)^{\prime} \frac{\mathcal{M}_{\alpha}^{1} \phi(z)}{z}\right) \\
& =\Re\left(\frac{\left(-z^{4}+2 z^{3}-6 z^{2}+2 z-1\right)\left(\frac{0.25 z}{(1-z)^{2}}+\frac{0.75 z}{(1+z)^{2}}\right)}{z\left(z^{2}-1\right)^{3}}\right) \\
& >0,
\end{aligned}
$$

when $z \rightarrow 1$. Hence, in view of Theorem $1, \frac{\mathcal{M}_{\alpha}^{1} \phi(z)}{z} \in \mathcal{P}(\epsilon)$ for some $\epsilon \in[0,1)$.

\section{Conclusions and Future Works}

Motivated by this method, in the recent investigation we have presented new classes of univalent functions that connect to a symmetric differential operator in the open unit disk. We have obtained sufficient and necessary conditions in relation to these subclasses. Linear combinations, operator and other properties are also explored. For further research, we indicate to study the certain new classes related to other types of analytic functions such as meromorphic, harmonic and $p$-valent functions with respect to symmetric points associated with SDO.

Author Contributions: Conceptualization, R.W.I. and M.D.; methodology, R.W.I.; validation, R.W.I. and M.D.; formal analysis, R.W.I. and M.D.; investigation, R.W.I. and M.D.; writing-original draft preparation, R.W.I.; writing-review and editing, M.D.

Funding: The second author is partially supported by Universiti Kebangsaan Malaysia grant: GUP-2017-064.

Acknowledgments: The author wishes to express his profound gratitude to the anonymous referee for his/her careful reading of the manuscript and the very useful comments that have been implemented in the final version of the manuscript. 
Conflicts of Interest: The authors declare no conflict of interest.

\section{References}

1. Duren, P. Univalent Functions; Grundlehren der mathematischen Wissenschaften; Springer: New York, NY, USA, 1983; Volume 259, ISBN 0-387-90795-5.

2. Sàlàgean, G.S. Subclasses of univalent functions, Complex Analysis-Fifth Romanian-Finnish Seminar, Part 1 (Bucharest, 1981). In Lecture Notes in Mathematics; Springer: Berlin, Germany, 1983; Volume 1013, pp. 362-372.

3. Al-Oboudi, F.M. On univalent functions defined by a generalized Sàlàgean operator. Int. J. Math. Math. Sci. 2004, 27, 1429-1436. [CrossRef]

4. Ibrahim, R.W.; Darus, M. Subordination inequalities of a new Sàlàgean difference operator. Int. J. Math. Comput. Sci. 2019, 14, 573-582.

5. Ovsyannikov, L.V. Group Analysis of Differential Equations; Academic Press: New York, NY, USA, 1982.

6. Sidorov, N.; Loginov, B.; Sinitsyn, A.V.; Falaleev, M.V. Lyapunov-Schmidt Methods in Nonlinear Analysis and Applications; Springer: New York, NY, USA, 2013.

7. Miller, S.S.; Mocanu, P.T. Differential Subordinations: Theory and Applications; CRC Press: Boca Raton, FL, USA, 2000.

8. Lupas, A.A. On special differential subordinations using Salagean and Ruscheweyh operators. Math. Inequal. Appl. 2009, 12, 781-790.

9. Arif, M.; Ahmad, K.; Liu, J.L.; Sokół, J. A new class of analytic functions associated with Sălăgean operator. J. Funct. Spaces 2019, 6157394. [CrossRef]

10. Sakaguchi, K. On a certain univalent mapping. J. Math. Soc. Jpn. 1959, 11, 72-75. [CrossRef]

11. Das, R.N.; Singh, P. On subclasses of schlicht mapping. Indian J. Pure Appl. Math. 1977, 8, 864-872.

(C) 2019 by the authors. Licensee MDPI, Basel, Switzerland. This article is an open access article distributed under the terms and conditions of the Creative Commons Attribution (CC BY) license (http://creativecommons.org/licenses/by/4.0/). 